\title{
Characterization of multi-drug tolerant persister cells in Streptococcus suis
}

\author{
Jörg Willenborg ${ }^{1 *}$, Daniela Willms ${ }^{1}$, Ralph Bertram², Ralph Goethe ${ }^{1}$ and Peter Valentin-Weigand ${ }^{1}$
}

\begin{abstract}
Background: Persister cells constitute a subpopulation of dormant cells within a microbial population which are genetically identical but phenotypically different to regular cells. Notably, persister cells show an elevated tolerance to antimicrobial agents. Thus, they are considered to represent a microbial 'bet-hedging' strategy and are of particular importance in pathogenic bacteria.

Results: We studied the ability of the zoonotic pathogen Streptococcus (S.) suis to form multi-drug tolerant variants and identified persister cells dependent on the initial bacterial growth phase. We observed lower numbers of persisters in exponential phase cultures than in stationary growth phase populations. S. suis persister cells showed a high tolerance to a variety of antibiotics, and the phenotype was not inherited as tested with four passages of S. suis populations. Furthermore, we provide evidence that the persister phenotype is related to expression of genes involved in general metabolic pathways since we found higher numbers of persister cells in a mutant strain defective in the catabolic arginine deiminase system as compared to its parental wild type strain. Finally, we observed persister cell formation also in other $S$. suis strains and pathogenic streptococcal species.
\end{abstract}

Conclusions: Taken together, this is the first study that reports multi-drug tolerant persister cells in the zoonotic pathogen S. suis.

Keywords: Streptococcus suis, Persister cells, Multidrug tolerance, Antibiotics

\section{Background}

Formation of persister cells by bacteria is a phenomenon that, amongst others, contributes to tolerance of a bacterial subpopulation to antimicrobial agents. Notably, this antibiotic tolerance of persister cells is distinct from genetically inherited resistance. The persister cell subpopulation has been firstly described and named nearly 70 years ago [1] and research on persister cells has identified a number of typical characteristics as debated recently [2]. Bacterial persister cells seem to represent a stage of dormancy that protects them from killing by antimicrobial substances, even in the presence of concentrations which vastly exceed the minimal inhibitory concentration (MIC). Persister cells are genetically identical to antibiotic sensitive bacteria within a population, but have a distinct phenotype in that they are tolerant to certain antibiotics [3]. Since most antibiotics target bacterial components or pathways involved

\footnotetext{
*Correspondence: joerg.willenborg@tiho-hannover.de

'Institute of Microbiology, University of Veterinary Medicine, Hannover, Germany

Full list of author information is available at the end of the article
}

in replication, the dormancy stage in persister cells is thought to be the underlying mechanism of antibiotic tolerance [4]. Nevertheless, persister celIs can switch from the dormant into a replicating stage. This 'bet-hedging' strategy is thought to be a survival strategy of microbial populations [5].

Two different types of persister cells have been postulated. Type I persister cells are formed in response to environmental stimuli, for instance during the initiation of the stationary growth phase, whereas type II persister cells arise stochastically within a dividing population [6,7].

A recent report proposed a 'persistence-if-stuff-happens' hypothesis, i.e. persister cell formation is an inevitable process due to cellular errors that produce transient states of reduced replication and/or metabolic activity in a single bacterium [8]. Nevertheless, in the last years many attempts have been made to identify molecular factors involved in the development of a persister cell subpopulation. There is increasing evidence that toxin-antitoxin modules, quorumsensing molecules, global transcriptional regulators, and 
molecules of the stringent response like (p)ppGpp are involved in persister cell formation [4,9-13].

Since the first report by Bigger in 1944 [1], bacterial persister cells have been described for a number of different species, including Escherichia coli [14], Staphylococcus aureus [14,15], Pseudomonas aeruginosa [16], and Mycobacterium tuberculosis $[17,18]$. For most of these bacterial species persister cells have also been found in biofilms, which contribute to recalcitrant and/or recurrent infections after antibiotic therapy [4,19-25].

Little is known about persister cell formation in streptococci $[9,26]$. Within pathogenic streptococci, the zoonosis Streptococcus suis (S. suis) is of particular interest since it can cause very severe diseases, such as sepsis, meningitis and streptococcal toxic shock like syndrome in humans who are in close contact to pigs or pig products [27-30]. Notably, S. suis has been shown to be one of the most frequent causes of adult bacterial meningitis in Asian countries including Vietnam and Thailand [31,32]. S. suis infections are widely distributed in pigs, but can also occur in wildlife animals such as wild rabbits or wild boars $[33,34]$. In pigs $S$. suis is a frequent early colonizer of the upper respiratory tract. In young pigs $S$. suis is also a major cause of meningitis, arthritis, and septicemia. Thus, S. suis infections are a major concern in the swine producing industry as they lead to high financial losses [35].

Since antibiotics are widely used to control S. suis infections (in humans and in animals), we examined the ability of $S$. suis to produce antibiotic tolerant persister cells. We analyzed the effects of the initial bacterial growth phase on persister cell formation, the tolerance of these cells to different types of antibiotics, as well as persister cell levels of different S. suis strains and other human pathogenic streptococci. Our results show for the first time that $S$. suis forms high levels of persister cells that confer tolerance to a variety of antimicrobial compounds. We also present evidence that persister cell formation is not only found in S. suis but also in other streptococcal species.

\section{Results}

\section{Identification of a multi-drug tolerant persister cell subpopulation in $S$. suis}

Since persister cell formation is important for antibiotic tolerance and recurrent infections, we studied the occurrence of persister cells in the zoonotic pathogen S. suis using a highly virulent serotype 2 strain, strain 10 . First we determined the minimal inhibitory concentration (MIC) of six antibiotics with different modes of action for exponential grown S. suis strain 10 by the standard microdilution assay (see Additional file 1: Table S1), because one main characteristic of persister cells is the ability to tolerate concentrations of different antimicrobial compounds above the MIC. Following, to test whether S. suis is capable of producing persister cells that tolerate antibiotic treatment, we performed antibiotic killing experiments with a 100fold MIC of each antimicrobial compound. Antibiotic challenge was performed with cultures grown either to exponential or stationary phase. Since a 100-fold MIC should inactivate antibiotic-sensitive normal growing bacteria, we assumed that this treatment would result in characteristic biphasic-killing characterized by an initial rapid killing of the bulk of the bacterial population followed by a distinct plateau of surviving drug tolerant persister cells [6]. As depicted in Figure 1A, gentamicin treatment of exponential grown S. suis resulted in decrease of bacterial CFU by three orders of magnitude within the first hour and a subsequent plateau phase in the following hours. When we applied $\beta$-lactam antibiotics and ciprofloxacin the killing was not as pronounced as observed for gentamicin, nevertheless a slow decrease of life counts was seen over time. Nearly no killing was observed after treatment with rifampicin. In contrast, daptomycin was able to completely kill the bacterial population without detectable survival of persister cells. These data indicate that within an exponential grown S. suis culture a subpopulation of antibiotic tolerant
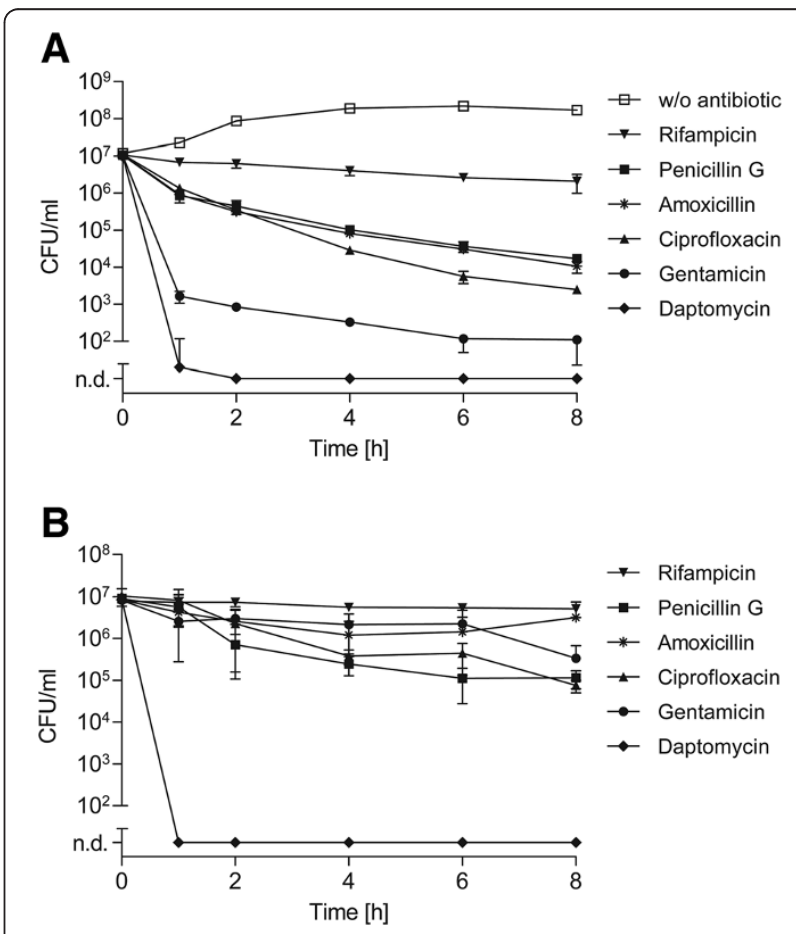

Figure 1 Killing kinetics of $S$. suis exposed to different antibiotics. Exponential (A) or stationary (B) grown S. suis strain 10 was treated with 100-fold MIC of indicated antibiotics over time. The limit of detection was defined as $100 \mathrm{CFU} / \mathrm{ml}$ throughout all killing experiments. All lower bacterial numbers were considered as not detectable (n. d.). The values are means of two biological replicates and error bars indicate the standard deviation. An untreated culture without any antibiotic challenge (w/o antibiotic) served as a control. 
persister cells exists, which show different degrees of tolerance depending on the class of antibiotic.

Next we studied the persister cell levels of stationary grown $S$. suis since for several other bacterial species a drastic increase in persister levels has been reported at the onset of stationary growth phase [4]. Antibiotic treatment of stationary cultures of S. suis with 100-fold MIC resulted in a substantial drug tolerance, i.e. a distinct biphasic killing pattern such as seen with exponential cultures was not observed (Figure 1A vs. B). Only a slight decrease in numbers of CFU was detected over time after treatment with $\beta$-lactams, ciprofloxacin and gentamicin. In the case of gentamicin a relative difference of approximately three logarithmic orders in CFU was recorded after the first hour of antibiotic treatment, when comparing populations of exponential and stationary grown S. suis. Notably, growth to the stationary growth phase did not enhance the tolerance of S. suis to the cyclic lipopeptide daptomycin which completely killed the $S$. suis population after only one hour of treatment. Taken together, the killing kinetics revealed that under the conditions tested S. suis develops a growth phase dependent subpopulation showing antibiotic tolerance to a variety of antimicrobial compounds except daptomycin.

The persister cell phenotype of $S$. suis is not inherited and dominated by type I persisters

In contrast to genetically encoded antimicrobial resistance, multidrug tolerance of persister cells is a transient and non-heritable phenotype $[10,26]$. To test heritability of antimicrobial tolerance, exponential grown $S$. suis was treated with 100-fold MIC of gentamicin and the surviving population was used to repeat a new cycle. Four consecutive cycles were tested. Gentamicin was selected for these experiments since this treatment resulted in pronounced biphasic killing curves in the first hours after antibiotic treatment. As depicted in Figure 2A, tolerance to gentamicin of the initial population was not transferred to following S. suis generations. The characteristic biphasic killing curve upon antibiotic treatment was observed irrespective of the number of passages. These results suggest that the formation of a S. suis persister cell subpopulation and antimicrobial tolerance is not inherited and of transient nature.

In order to dissect whether type I or type II persisters are responsible for gentamicin tolerance, we performed a persister cell elimination assay. In this assay the formation of type I persisters is suppressed by sequential re-inoculation of an early exponential culture. This procedure leads to dilution of type I persisters whilst stochastically build type II persister cell levels should remain constant. As depicted in Figure $2 \mathrm{~B}$ the percentage of antibiotic tolerant persisters decreased sequentially after 100 -fold MIC gentamicin challenge when the bacterial culture was kept in the early growth phase for three cycles. This data indicate that gentamicin tolerant persisters are not or only rarely produced in the early exponential growth phase and that most of the tolerant bacteria represented type I persisters. These were probably 'left overs' from the overnight culture and became diluted within repeated cycles of exponential growth.

\section{S. suis persister cells also tolerate combinations of different antibiotics}

Antibiotics like penicillin are frequently used to treat $S$. suis infections, sometimes in combination with other antibiotics like aminoglycosides. However, relapses of $S$. suis infections in pigs and humans have been reported [36]. Furthermore, penicillin and gentamicin are widely used in standard antibiotic protection assays to quantify intracellular bacteria in in vitro cell culture experiments.
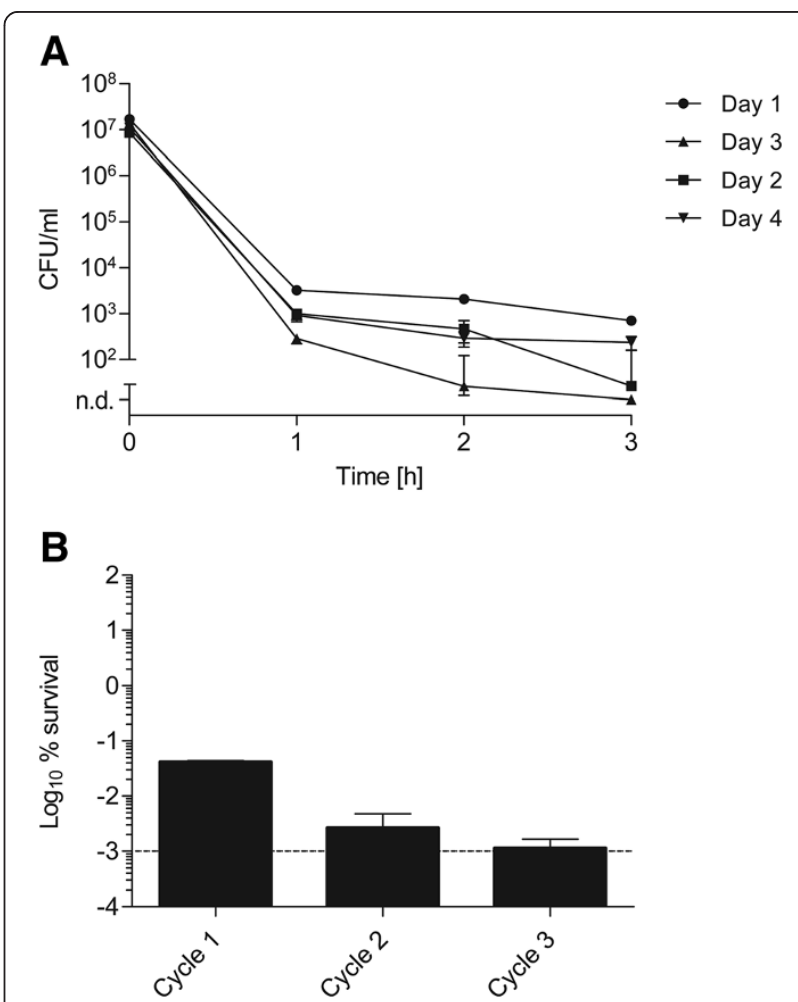

Figure 2 Test for the heritability of persistence and elimination of persister cells. (A) Exponential grown S. suis strain 10 was treated with 100-fold MIC of gentamicin for three hours, and at indicated time points CFU were determined. Subsequently, surviving bacteria were incubated in fresh THB media overnight, then grown to early logarithmic phase and challenged with 100-fold MIC of gentamicin. This procedure was repeated for four consecutive cycles. The values are means of three biological replicates and error bars indicate the standard deviation.

(B) S. suis strain 10 was sequentially grown to early exponential growth phase. At each cycle CFU of the initial inoculum and of surviving bacteria after a one-hour 100-fold MIC gentamicin challenge were determined. Data were expressed for each cycle as percentage of surviving bacteria in relation to the initial inoculum before antibiotic treatment. The dotted line represents the limit of detection. Standard deviation is shown for three replicates. 
Therefore, we investigated S. suis tolerance against a combination of penicillin (200-fold MIC) and gentamicin (4fold MIC) that correspond to the concentrations applied in these antibiotic protection experiments. After simultaneous treatment of exponential grown $S$. suis with penicillin and gentamicin we observed a biphasic killing curve characterized by a rapid decrease of CFU numbers within the first hour and a subsequent plateau of surviving bacteria persisting for more than 8 hours (Figure 3A). The killing kinetics of stationary grown bacteria treated similarly resembled treatment with gentamicin alone, as depicted in Figure 1B. Similar to what we observed after treatment with a single antibiotic, the tolerance to a combination of penicillin and gentamicin was not inherited, as revealed from heritability tests (Figure 3B). These data suggest that S. suis persister cells are capable of tolerating not only single antibiotics, but also a combination of penicillin and aminoglycosides.

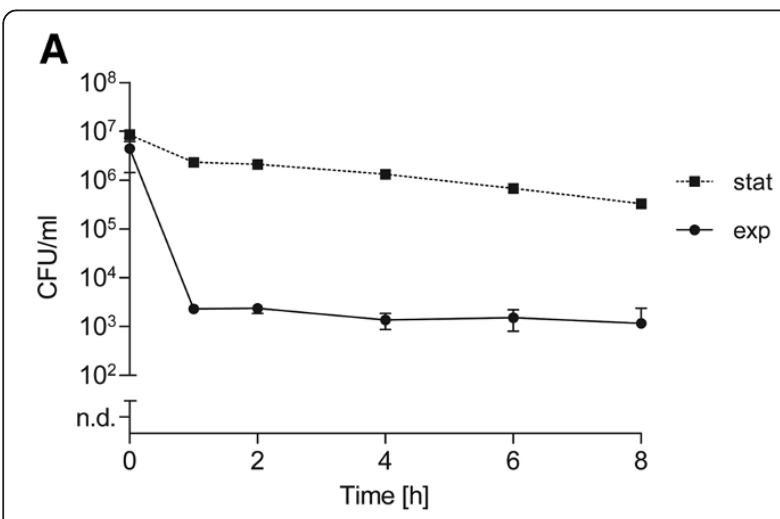

B

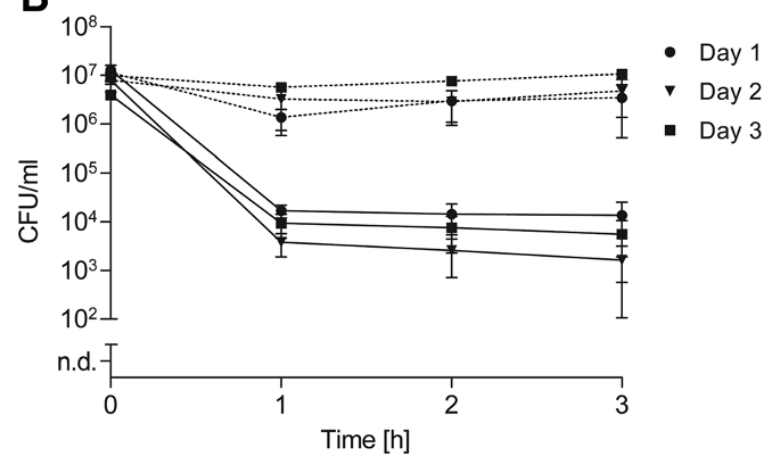

Figure 3 Time-dependent killing after combined antibiotic treatment. (A) Exponential (solid line) and stationary (dotted line) grown S. suis strain 10 was exposed to a combined antibiotic treatment of 200-fold MIC of penicillin and 4-fold MIC of gentamicin over time. (B) This penicillin/gentamicin combination was also used in a heritability test with exponential (solid line) and stationary (dotted line) grown S. suis in three consecutive cycles. The values are means of two biological replicates plated in triplicate. Error bars indicate the standard deviation.
Persister cell formation in S. suis is affected by the global transcriptional regulator $\mathrm{CcpA}$ and the catabolic arginine deiminase system

Several genes and gene products have been implicated in antibiotic tolerance, like toxin-antitoxin (TA) modules, quorum-sensing molecules and global transcriptional regulators $[4,9]$. In previous studies we have shown that CcpA is a pleiotropic regulator of $S$. suis carbon metabolism, virulence gene expression and the expression of the arginine deiminase $(A D)$ system [37-39]. The latter is crucial for bacterial survival in acidic environments and is most likely required for alternative ATP generation. Hence, we tested respective $S$. suis mutant strains $10 \Delta c c p \mathrm{~A}$ and $10 \triangle \mathrm{AD}$ for gentamicin tolerant persister cells. CFU of bacterial strains grown to the exponential growth phase were determined over time after treatment with 100fold MIC gentamicin. The gentamicin MIC values of the mutant strains did not differ from those of the wild type strain. No change in persister levels was observed for exponential grown strain $10 \Delta c c p \mathrm{~A}$, whereas the $\mathrm{AD}$ mutant strain $10 \triangle \mathrm{AD}$ showed an approximately two log-fold higher persister cell level over time compared to the wild type (Figure 4A). This difference was abrogated when stationary growth phase cultures were challenged by gentamicin (Figure 4B). Interestingly, during the later growth phase the persister level of strain $10 \Delta c c p \mathrm{~A}$ decreased as compared to the wild type and strain $10 \Delta \mathrm{AD}$.

\section{Persister cell formation occurs in different S. suis strains and streptococcal species}

Next, we tested antibiotic tolerance and persister cell formation in other S. suis strains and streptococcal species. For this, we analyzed a human serotype 2 isolate (strain 05ZYH33) originating from a $S$. suis outbreak in China and a serotype 9 strain (strain A3286/94) isolated from a pig with meningitis [40,41]. The MIC values of gentamicin for strain 05ZYH33 and strain A3286/94 are given in Additional file 1: Table S1. In all strains, treatment with 100-fold MIC of gentamicin induced the characteristic biphasic killing curve and resulted in a complete killing of bacteria after 24 hours. No substantial differences could be observed between strains in the exponential growth phase (Figure 5). On the other hand, using stationary cultures strain 10 showed the highest degree of drug tolerance. Strains A3286/94 and 05ZYH33 were killed more efficiently, especially during the first hour of antibiotic treatment, with persister cell differences of up to two logfold CFU. After 24 hours of gentamicin treatment viable bacteria could only be recovered in strain 10, but not in strains A3286/94 and 05ZYH33, respectively (data not shown). These data indicate that various $S$. suis strains and serotypes form persisters with different frequencies and antibiotic tolerance characteristics. 


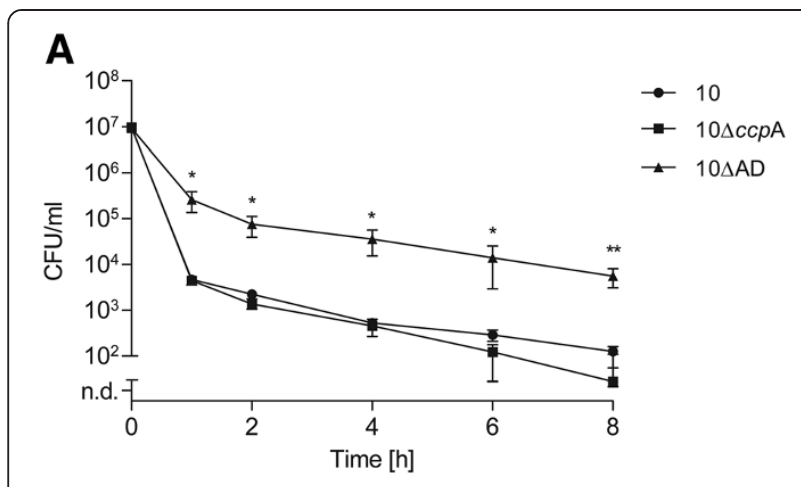

B

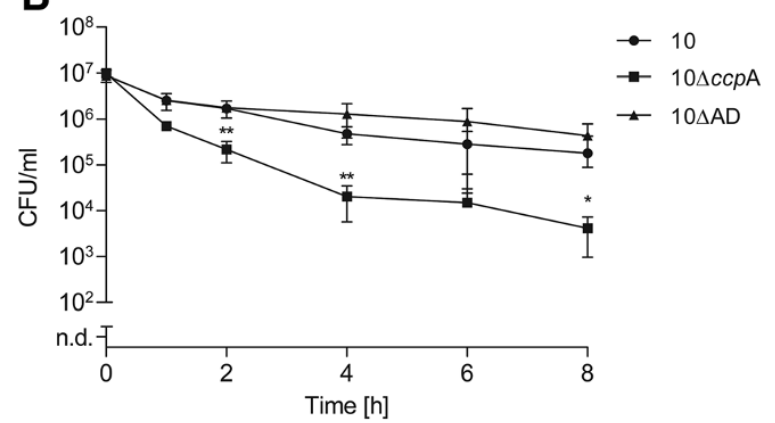

Figure 4 Effect of specific gene inactivation on S. suis persister formation. Exponential (A) or stationary (B) grown S. suis strains were treated with 100-fold MIC of gentamicin over time. Persister cell levels were determined for the wild type strain 10, and its knock-out mutant strains $10 \triangle c c p A$ and $10 \triangle A D$, which lack the genes coding for the global transcriptional regulator CcpA and the catabolic arginine deiminase system, respectively. The values are means of three biological replicates and error bars indicate the standard deviation. Significant differences to wildtype persister levels were calculated by a one-tailed $t$-test $\left({ }^{*}, P<0.05 ;{ }^{*}, P<0.01\right)$.

Since antibiotic tolerance has been reported for other streptococcal species [42-44] we studied persister cell formation in selected strains of other streptococci, including S. pyogenes, S. agalactiae, and S. gordonii after treatment with 100-fold MIC gentamicin. The determined MIC values for each strain are listed in Additional file 1: Table S1. Interestingly, in contrast to S. suis neither exponential nor stationary grown streptococci of the tested strains displayed a gentamicin tolerant subpopulation (data not shown). Notably, we could not detect any gentamicin tolerant subpopulation for S. pyogenes, S. gordonii, and $S$. agalactiae overnight cultures as shown in Figure 6A. On the other hand, treatment with 100-fold MIC of ciprofloxacin resulted in a drug-specific tolerance for at least 8 hours (Figure 6B). The proportion of ciprofloxacin tolerant bacteria was higher for S. suis strain 10 and $S$. pyogenes strain A40 as compared to the other streptococcal species. These data indicate that drug tolerant subpopulations might also occur in other streptococcal species, but the extent of tolerance seems to vary between different antibiotics.
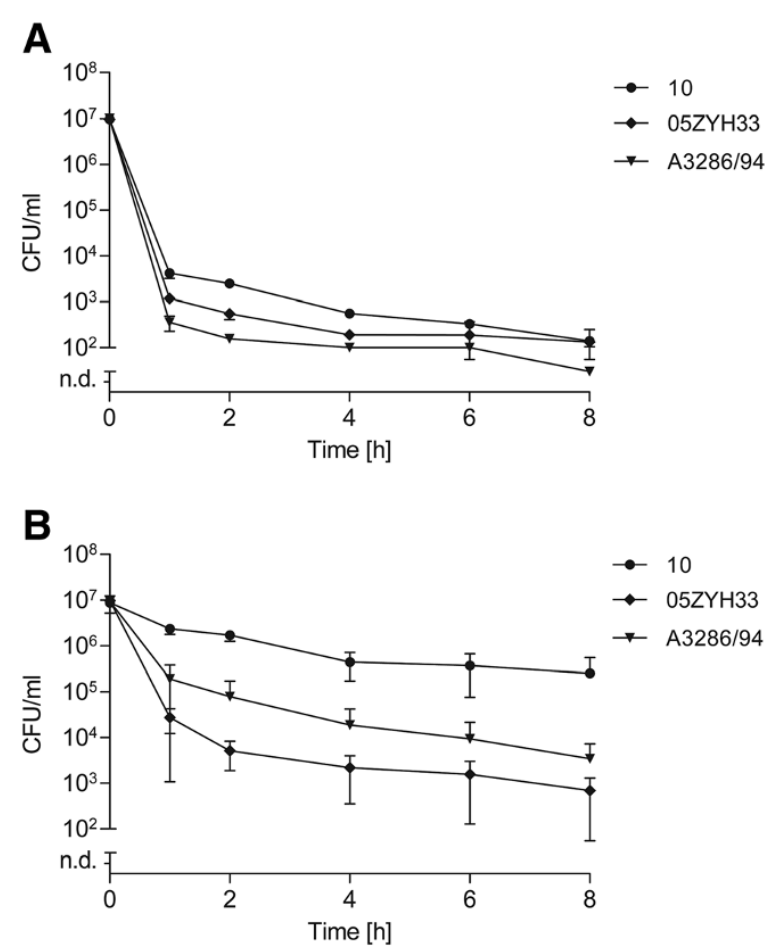

Figure 5 Persister cell levels of different $S$. suis strains.

Exponential (A) or stationary (B) grown S. suis strains were treated with 100-fold MIC of gentamicin over time. Persister cell levels were determined for the porcine serotype 2 isolate strain 10, a porcine serotype 9 isolate strain A3286/94, and a human serotype 2 isolate strain 05ZYH33. The values are means of two biological replicates and error bars indicate the standard deviation.

\section{Discussion}

Generation of bacterial persister cells is important not only with respect to the understanding of population dynamics but also concerning antibiotic tolerance in respective therapy of infections [45]. Accordingly, there is growing evidence that bacterial persisters are involved in relapses of refractory bacterial infections and in the establishment of resistance mechanisms in bacteria [21]. Owing to this it seems not surprising that persister cells have been described for numerous pathogenic bacteria. In this study we have shown for the first time that $S$. suis forms multi-drug tolerant persister cells. Even though 100-fold MIC is unlikely to be achieved in therapy of natural infections, we assumed that this treatment would be a method of choice to identify highly drug-tolerant persister cells of S. suis in accordance to results reported for S. aureus [15]. By this we identified persister cell formation in three different $S$. suis strains, suggesting that this phenomenon may be a general trait among this species. Though this has to be further confirmed by testing more $S$. suis strains and antibiotics that are of higher clinical relevance to treat S. suis infections in pigs and humans, persister cells should be considered in the future in cases of 


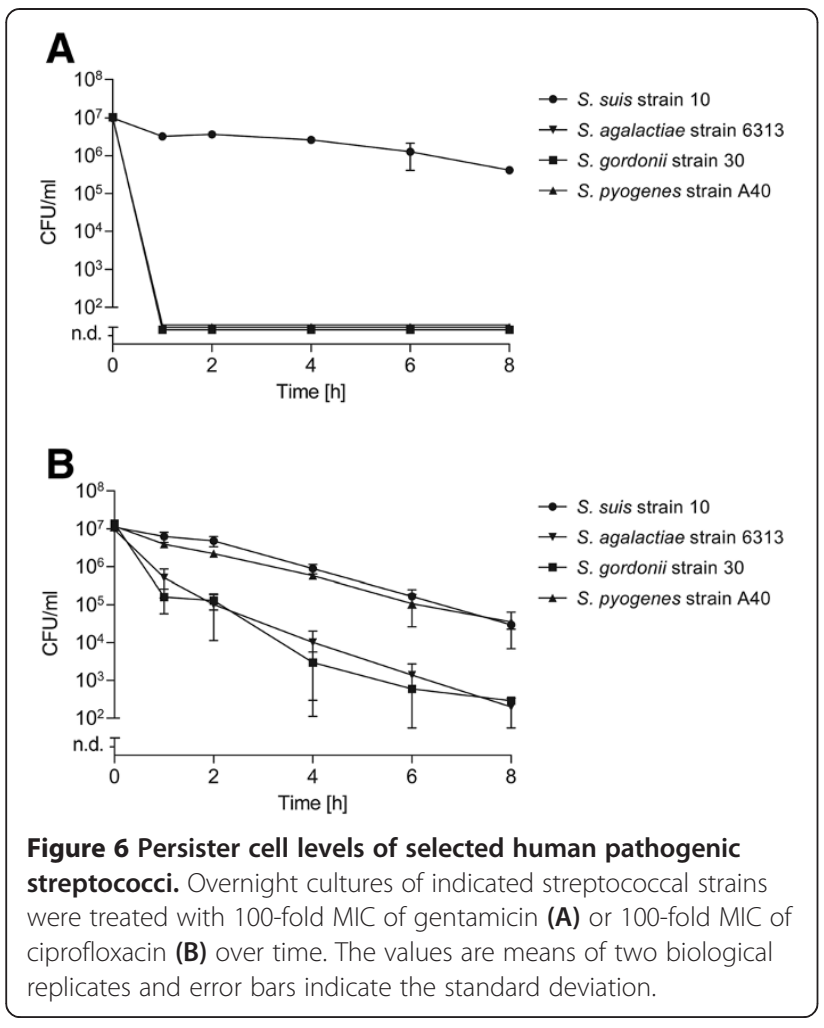

ineffective antibiotic treatments or when studying antibiotic tolerance of S. suis.

In line with several previous studies $[3,14,22,46]$ the number of persisters observed was higher during stationary growth of S. suis when compared to exponential grown bacteria. Type I persisters were found to be the main source of antibiotic tolerance in our experiments. Among other stress signals, nutrient limitation in stationary growth is thought to be a trigger inducing down-regulation of the metabolic activity and bacterial dormancy in energydeprived cells which can protect the bacteria from antibiotic killing. We found some hints for involvement of the catabolic enzyme system ADS, since approximately two log-fold higher levels of persister cells were found in the exponential growth phase of an arginine deiminase knockout strain $(10 \Delta \mathrm{AD})$ as compared to its wild type strain. In S. suis the arginine deiminase system metabolizes arginine as a substrate to produce energy in form of ATP [38]. The diminished ATP levels may lead to reduced general metabolic activity of strain $10 \triangle \mathrm{AD}$ that might explain the slower growth rate (see Additional file 2: Figure S1) and enhanced number of antibiotic tolerant persister cells. Furthermore, the $c c p \mathrm{~A}$ deficient strain exhibited lower numbers of persister cells in the stationary growth phase when compared to the wild type. This is in agreement with studies in S. gordonii showing that a ccpA knock-out resulted in an increased sensitivity of the bacteria to penicillin treatment [47]. Since CcpA is a pleiotropic regulator that is important for a balanced metabolic flux in the central carbon metabolism, the alteration of central metabolic processes may influence persister cell formation of S. suis. Accordingly, an interplay between carbohydrate consumption and formation of persisters has recently been demonstrated for E. coli [12]. Further studies are needed to clarify the mechanisms involved in CcpA and/or arginine deiminase dependent changes in antibiotic tolerance of S. suis.

When using antibiotics with varying modes of action, resulting killing profiles were quite different, ranging from pronounced biphasic killing patterns to nearly plane curves, at least for exponential grown $S$. suis. These findings seem to be highly dependent on the type of antibiotic used, which is also emphasized by the fact that treatment with the $\beta$-lactam antibiotics amoxicillin and penicillin resulted in similar killing curves. Thus we speculated a common mechanism of tolerance for certain antibiotics and tested the gentamicin tolerance in other streptococcal species. S. suis strain 10 highly tolerated 100-fold MIC of gentamicin, whereas the other streptococcal strains were completely killed after one hour. These data suggest that a specific mechanism for gentamicin tolerance of $S$. suis persisters may have evolved and that this is, most likely, not due to a shared genetic background within the genus Streptococcus. Interestingly, after gentamicin treatment of S. suis we also observed a small-colony-variant (SCV) like phenotype (data not shown) that has also been reported for S. aureus upon aminoglycoside treatment [15,48]. Although it reverted to the typical large-colony phenotype after subcultivation, it remains to be elucidated if this phenotype will change to a stable phenotype after longer exposure times and altered antibiotic tolerance to aminoglycosides. However, at the stationary growth phase the investigated $S$. suis strain 10 highly tolerated several antimicrobials targeting different bacterial components over time. Given the high rate of multi-drug tolerant cells produced by S. suis strain 10 during stationary growth, it was remarkable that the cyclic lipopeptide daptomycin efficiently eradicated this subpopulation. This is in contrast to observations that in S. aureus 100-fold MIC of daptomycin failed to eradicate stationary phase cultures [15]. Even though the MIC for daptomycin is rather high when compared to that of other streptococcal species [49] this treatment eradicated S. suis persister cells in vitro.

In the last years bacterial persistence and enhanced antibiotic tolerance was intensively discussed in the context of recurrent infections caused by bacterial pathogens. Interestingly, a human case of recurrent septic shock due to a S. suis serotype 2 infection has previously been reported [50]. Together with our present study this suggests a clinical relevance of $S$. suis persisters. Although experimental evidence for $S$. suis persister cell and biofilm formation in vivo is yet missing, S. suis is able to produce biofilms in vitro that tolerate antibiotic challenge [51,52]. Given 
the fact that the S. suis colonization rate of pigs is nearly $100 \%[35,53,54]$ and that antibiotic treatment with penicillin, ampicillin, or ceftiofur failed to eliminate the tonsillar carrier state of S. suis in swine [55], it is plausible to speculate that persister cells, possibly also as part of biofilm structures, may contribute to the observed problems in antibiotic treatments. Indeed, $P$. aeruginosa persister cells have been described as the dominant population responsible for drug tolerance in biofilms [22].

\section{Conclusions}

Our study showed that the zoonotic pathogen S. suis is able to form a multi-drug tolerant persister cell subpopulation. S. suis persister cells tolerated a variety of antimicrobial compounds that were applied at 100 -fold of MIC and could be detected in different $S$. suis strains. Thus, our study provides a basis for future studies on the role of S. suis persister cells in bacterial colonization of host tissues, general antibiotic tolerance, and recurrent infections.

\section{Methods}

Bacterial strains, media, and growth conditions

All bacterial strains investigated in this study (listed in Table 1) were grown in complex Todd Hewitt Broth (THB, Becton Dickinson Diagnostics) medium at $37^{\circ} \mathrm{C}$. If not stated otherwise cryo-conserved bacterial stocks were used in the experiments. Preliminary experiments with cryo-conserved and freshly prepared bacterial cultures had revealed no significant differences in persister cell formation assays (data not shown), similar to what has been reported for E. coli [6]. For the preparation of bacterial stocks, overnight cultures were diluted to an optical density at $600 \mathrm{~nm}\left(\mathrm{OD}_{600}\right)$ of 0.02 in fresh THB medium and further incubated until bacteria reached

\section{Table 1 Bacterial strains used in this study}

\begin{tabular}{llc}
\hline Strain & Description & Reference \\
\hline S. suis & & \\
10 & Virulent serotype 2 strain, porcine isolate & {$[56]$} \\
$10 \triangle c c p A$ & Strain 10 ccpA mutant; ccpA::Em ${ }^{R}$ & {$[39]$} \\
$10 \triangle \mathrm{AD}$ & $\begin{array}{l}\text { Strain } 10 \text { arginine deiminase operon } \\
\text { mutant; } \text { arcA::Spc }\end{array}$ & {$[38]$} \\
05ZYH33 & $\begin{array}{l}\text { Virulent serotype 2 strain, isolate from } \\
\text { human outbreak in China }\end{array}$ & {$[40]$} \\
A3286/94 & Virulent serotype 9 strain, porcine isolate & {$[41]$} \\
S. agalactiae & & \\
6313 & A clinical isolate belonging to serotype III & {$[57]$} \\
S. gordonii & & \\
30 & & {$[58]$} \\
S. pyogenes & & \\
A40 & A clinical isolate belonging to M type 12 & {$[59]$} \\
\hline
\end{tabular}

either the early exponential (exp) or stationary (stat) growth phase as depicted in Additional file 2: Figure S1. Then $19 \mathrm{ml}$ of exponential grown or $4 \mathrm{ml}$ of stationary grown bacterial cultures were collected and centrifuged at $4000 \times g$ for $10 \mathrm{~min}$ at $4^{\circ} \mathrm{C}$. Bacterial pellets were washed once in phosphate-buffered saline, resuspended in THB medium containing 15\% glycerol $(\mathrm{v} / \mathrm{v})$, and aliquots were immediately shock frozen in liquid nitrogen. Frozen cultures were kept at $-80^{\circ} \mathrm{C}$ until use and numbers of viable cells were determined by serial plating on sheep blood Columbia agar plates. All antibiotic treatments were performed in chemically defined medium, RPMI 1640 (Life Technologies), which is routinely used in cell culture.

\section{Antibiotics and determination of minimal inhibitory concentration (MIC)}

Daptomycin (commercial Cubicin ${ }^{\circ}$ ) analytic grade powder was purchased from Novartis Pharma. Penicillin G, ciprofloxacin, amoxicillin, and rifampicin were purchased from Sigma, and gentamicin from Roth. The antimicrobial solutions were prepared freshly prior to each application according to the manufacturers' recommendations.

The MIC of each antibiotic was determined in duplicate by the microdilution technique in 96-well plates. Serial two-fold dilutions of different antibiotics prepared in RPMI 1640 medium were inoculated each with $5 \times 10^{5}$ colony forming units (CFU) of exponential grown cryoconserved bacteria per well. MICs were also determined for freshly prepared bacterial cultures and did not significantly differ from MICs of cryo-conserved bacteria (data not shown). Plates were covered with a Breathe-Easy ${ }^{\circ}$ sealing membrane to avoid evaporation and incubated for 24 hours at $37^{\circ} \mathrm{C}$. The lowest antibiotic concentration that inhibited visible bacterial growth was defined the MIC. The determined MIC values are listed in Additional file 1: Table S1.

\section{Test for persister cell formation}

Chemically defined RPMI 1640 medium was inoculated with $1 \times 10^{7} \mathrm{CFU}$ of either exponential or stationary grown cryo-conserved bacteria. Freshly prepared antimicrobial substances were added at a final concentration of 100-fold MIC, if not stated otherwise. Suspensions were incubated with end-over-end rotation at $37^{\circ} \mathrm{C}$. Samples were taken after 1, 2, 4, 6, and 8 hours for determination of CFU by serial dilution and plating. For this $100 \mu \mathrm{l}$ of bacterial suspensions were immediately harvested by centrifugation, once washed in sterile $0.85 \% \mathrm{NaCl}$ solution and spotted as $10 \mu \mathrm{l}$ aliquots on sheep blood Columbia agar plates in serial dilutions. Plating of the aliquots was performed in triplicates and all antibiotic killing experiments were performed at least with two biological replicates. Bacterial colonies were counted 24 and 48 hours 
after incubation at $37^{\circ} \mathrm{C}$ to ensure detection of slow growing bacteria. The results were analyzed with the GraphPad Prism 5 software and expressed in CFU/ml on a logarithmic scale. The limit of detection was defined as $100 \mathrm{CFU} /$ $\mathrm{ml}$ and lower bacterial numbers were considered not detectable (n. d.). If indicated statistical significance was determined by one-sided Student $t$ test.

\section{Heritability of persistence}

An overnight culture was diluted to an $\mathrm{OD}_{600}$ of 0.02 in fresh THB medium and further incubated until the early exponential growth phase was reached. Then bacteria were harvested by centrifugation, once washed with PBS, and inoculated in fresh RPMI medium containing 100fold MIC of the respective antibiotic to a final bacterial concentration of $1 \times 10^{7} \mathrm{CFU} / \mathrm{ml}$. The suspensions were incubated at $37^{\circ} \mathrm{C}$ with moderate end-over-end rotation. Samples were taken hourly as indicated and the CFUs were determined after removal of remaining antibiotics by washings as described above. After 3 hours of antibiotic treatment (surviving) bacteria were collected by centrifugation, once washed in PBS, inoculated in fresh THB medium and grown overnight. This culture was then used to start a new cycle of antibiotic treatment with exponential grown bacteria. This procedure was repeated with three consecutive cycles and the experiment performed at least with two biological replicates. Colonies were counted and CFUs calculated as described above.

\section{Test for persister cell elimination}

To dissect whether the antibiotic tolerant persister population of S. suis strain 10 comprises type I or type II persister cells, we performed a persister cell elimination test as described by Keren et al. [14], with some modifications. Briefly, an overnight culture of S. suis strain 10 was adjusted to $\mathrm{OD}_{600}=0.02$ in fresh $\mathrm{THB}$ medium and further incubated until bacteria reached $\mathrm{OD}_{600}=0.2$. Then, aliquots of this culture were used to inoculate fresh THB medium to $\mathrm{OD}_{600}=0.02$ for a further cycle and to determine persister cell levels after a 100-fold MIC gentamicin challenge. A gentamicin challenge was done as described for the test of heritability of persistence with the exception that the antibiotic treatment lasted one hour. Dilutiongrowth cycles with subsequent antibiotic challenge were repeated thrice. For each cycle the initial inoculum before and the surviving bacteria after antibiotic challenge were determined by CFU counting. Data were expressed as percentage of surviving bacteria in relation to the initial inoculum before antibiotic treatment.

\section{Additional files}

Additional file 1: Table S1. MIC values of antimicrobial compounds $(\mu \mathrm{g} / \mathrm{ml})$ for different streptococcal strains. ND stands for 'not determined'.
Additional file 2: Figure S1. Growth kinetics of selected S. suis strains, isogenic mutants of S. suis strain 10, and strains of other streptococcal species in THB medium. For antibiotic tolerance assays bacteria were grown in complex THB medium and harvested at an $\mathrm{OD}_{600 \mathrm{~nm}}$ of 0.2 , reflecting the early exponential growth phase, or at the stationary growth phase of each strain that is indicated by a red coloured symbol in the graph. (A) Growth curves of selected S. suis strains and isogenic mutants of S. suis strain 10. (B) Growth curves of selected strains of other streptococcal species.

\section{Competing interests}

The authors declare that they have no competing interests.

\section{Authors' contributions}

JW and DW carried out the experiments and analyzed the data. RB helped with the design of the study and draft of the manuscript. JW, RG and PWW conceived the study, participated in its design and coordination and helped to draft the manuscript. All authors read and approved the final manuscript.

\section{Acknowledgements}

This work was supported by the Deutsche Forschungsgemeinschaft (DFG, Germany) as part of the Priority Programme SPP1316 (grants G0983-3/1 and BE4038/2-2). We gratefully acknowledge the following researchers for providing bacterial strains or antibiotics: Hilde Smith (Central Veterinary Institute, Wageningen University, Lelystad; S. suis strain 10), Susanne Talay (Helmholtz Centre for Infection Research, Braunschweig; S. pyogenes strain A40), Christoph Baums (University of Veterinary Medicine, Institute of Microbiology, Hannover; S. suis strain A3286/94), Jiaqi Tang (Research Institute for Medicine of Nanjing Command, Nanjing; S. suis strain 05ZYH33), and Mathias Hornef (Hannover Medical School, Hannover; Daptomycin/(Cubicin ${ }^{\oplus}$.

\section{Author details}

${ }^{1}$ Institute of Microbiology, University of Veterinary Medicine, Hannover, Germany. ${ }^{2}$ Department of Microbial Genetics, University of Tübingen, Tübingen, Germany.

Received: 25 February 2014 Accepted: 6 May 2014

Published: 12 May 2014

\section{References}

1. Bigger J: Treatment of staphylococcal infections with penicillin by intermittent sterilisation. Lancet 1944, 244(6320):497-500.

2. Balaban NQ, Gerdes K, Lewis K, McKinney JD: A problem of persistence: still more questions than answers? Nat Rev Microbiol 2013, 11:587-591.

3. Wiuff C, Zappala RM, Regoes RR, Garner KN, Baquero F, Levin BR: Phenotypic tolerance: antibiotic enrichment of noninherited resistance in bacterial populations. Antimicrob Agents Chemother 2005, 49:1483-1494.

4. Lewis K: Persister cells. Annu Rev Microbiol 2010, 64:357-372.

5. Kussell E, Leibler S: Phenotypic diversity, population growth, and information in fluctuating environments. Science 2005, 309:2075-2078.

6. Balaban NQ, Merrin J, Chait R, Kowalik L, Leibler S: Bacterial persistence as a phenotypic switch. Science 2004, 305:1622-1625.

7. Dhar N, McKinney JD: Microbial phenotypic heterogeneity and antibiotic tolerance. Curr Opin Microbiol 2007, 10:30-38.

8. Johnson PJ, Levin BR: Pharmacodynamics, population dynamics, and the evolution of persistence in Staphylococcus aureus. PLoS Genet 2013, 9:e1003123.

9. Fauvart M, De Groote VN, Michiels J: Role of persister cells in chronic infections: clinical relevance and perspectives on anti-persister therapies. J Med Microbiol 2011, 60:699-709.

10. Moyed HS, Bertrand KP: hipA, a newly recognized gene of Escherichia coli K-12 that affects frequency of persistence after inhibition of murein synthesis. J Bacteriol 1983, 155:768-775.

11. Gerdes K, Maisonneuve E: Bacterial persistence and toxin-antitoxin loci. Annu Rev Microbiol 2012, 66:103-123.

12. Amato SM, Orman MA, Brynildsen MP: Metabolic control of persister formation in Escherichia coli. Mol Cell 2013, 50:475-487.

13. Nguyen D, Joshi-Datar A, Lepine F, Bauerle E, Olakanmi O, Beer K, McKay G, Siehnel R, Schafhauser J, Wang Y, Britigan BE, Singh PK: Active starvation 
responses mediate antibiotic tolerance in biofilms and nutrient-limited bacteria. Science 2011, 334:982-986

14. Keren I, Kaldalu N, Spoering A, Wang Y, Lewis K: Persister cells and tolerance to antimicrobials. FEMS Microbiol Lett 2004, 230:13-18.

15. Lechner S, Lewis K, Bertram R: Staphylococcus aureus persisters tolerant to bactericidal antibiotics. J Mol Microbiol Biotechnol 2012, 22:235-244.

16. Brooun A, Liu S, Lewis K: A dose-response study of antibiotic resistance in Pseudomonas aeruginosa biofilms. Antimicrob Agents Chemother 2000, 44:640-646.

17. Keren I, Minami S, Rubin E, Lewis K: Characterization and transcriptome analysis of Mycobacterium tuberculosis persisters. MBio 2011, 2:e00100-e00111.

18. Wakamoto Y, Dhar N, Chait R, Schneider K, Signorino-Gelo F, Leibler S, McKinney JD: Dynamic persistence of antibiotic-stressed mycobacteria. Science 2013, 339:91-95

19. Shapiro JA, Nguyen VL, Chamberlain NR: Evidence for persisters in Staphylococcus epidermidis RP62a planktonic cultures and biofilms. J Med Microbiol 2011, 60:950-960.

20. Singh R, Ray P, Das A, Sharma M: Role of persisters and small-colony variants in antibiotic resistance of planktonic and biofilm-associated Staphylococcus aureus: an in vitro study. J Med Microbio/ 2009, 58:1067-1073.

21. Cohen NR, Lobritz MA, Collins JJ: Microbial persistence and the road to drug resistance. Cell Host Microbe 2013, 13:632-642.

22. Spoering AL, Lewis K: Biofilms and planktonic cells of Pseudomonas aeruginosa have similar resistance to killing by antimicrobials. J Bacterio 2001, 183:6746-6751.

23. Lewis K: Persister cells, dormancy and infectious disease. Nat Rev Microbiol 2007, 5:48-56.

24. Keren I, Shah D, Spoering A, Kaldalu N, Lewis K: Specialized persister cells and the mechanism of multidrug tolerance in Escherichia coli. J Bacteriol 2004, 186:8172-8180.

25. Lewis K: Multidrug tolerance of biofilms and persister cells. Curr Top Microbiol Immunol 2008, 322:107-131.

26. Leung $V$, Levesque CM: A stress-inducible quorum-sensing peptide mediates the formation of persister cells with noninherited multidrug tolerance. J Bacterio/ 2012, 194:2265-2274

27. Arends JP, Zanen HC: Meningitis Caused by Streptococcus suis in Humans. Rev Infect Dis 1988, 10:131-137.

28. Chanter $\mathrm{N}$, Jones PW, Alexander TJ: Meningitis in pigs caused by Streptococcus suis - a speculative review. Vet Microbiol 1993, 36:39-55.

29. Clifton-Hadley FA, Alexander TJ: The carrier site and carrier rate of Streptococcus suis type II in pigs. Vet Rec 1980, 107:40-41.

30. Gottschalk M, Xu J, Calzas C, Segura M: Streptococcus suis: a new emerging or an old neglected zoonotic pathogen? Future Microbiol 2010, 5:371-391.

31. Mai NT, Hoa NT, Nga TV, Linh LD, Chau TT, Sinh DX, Phu NH, Chuong LV, Diep TS, Campbell J, Nghia HD, Minh TN, Chau NV, de Jong MD, Chinh NT, Hien TT, Farrar J, Schultsz C: Streptococcus suis meningitis in adults in Vietnam. Clin Infect Dis 2008, 46:659-667.

32. Wertheim HF, Nguyen HN, Taylor W, Lien TT, Ngo HT, Nguyen TQ, Nguyen BN, Nguyen HH, Nguyen HM, Nguyen CT, Dao TT, Nguyen TV, Fox A, Farrar J, Schultsz C, Nguyen HD, Nguyen KV, Horby P: Streptococcus suis, an important cause of adult bacterial meningitis in northern Vietnam. PLoS One 2009, 4:e5973.

33. Baums CG, Verkuhlen GJ, Rehm T, Silva LM, Beyerbach M, Pohlmeyer K Valentin-Weigand P: Prevalence of Streptococcus suis genotypes in wild boars of Northwestern Germany. Appl Environ Microbiol 2007, 73:711-717.

34. Sanchez DR V, Fernandez-Garayzabal JF, Briones V, Iriso A, Dominguez L, Gottschalk M, Vela Al: Genetic analysis of Streptococcus suis isolates from wild rabbits. Vet Microbiol 2013, 165:483-486.

35. Varela NP, Gadbois P, Thibault C, Gottschalk M, Dick P, Wilson J: Antimicrobial resistance and prudent drug use for Streptococcus suis. Anim Health Res Rev 2013, 14:68-77.

36. Tan $\mathrm{H}$, Yeh $\mathrm{Bl}$, Seet CS: Deafness due to haemorrhagic labyrinthitis and a review of relapses in Streptococcus suis meningitis. Singapore Med J 2010, 51:e30-e33.

37. Fulde M, Willenborg J, De Greeff A, Benga L, Smith HE, Valentin-Weigand $P$, Goethe R: ArgR is an essential local transcriptional regulator of the arcABC operon in Streptococcus suis and is crucial for biological fitness in an acidic environment. Microbiology 2011, 157:572-582.

38. Gruening P, Fulde M, Valentin-Weigand P, Goethe R: Structure, regulation, and putative function of the arginine deiminase system of Streptococcus suis. J Bacterio/ 2006, 188:361-369.
39. Willenborg J, Fulde M, De Greeff A, Rohde M, Smith HE, Valentin-Weigand $P$, Goethe R: Role of glucose and CcpA in capsule expression and virulence of Streptococcus suis. Microbiology 2011, 157:1823-1833.

40. Chen C, Tang J, Dong W, Wang C, Feng Y, Wang J, Zheng F, Pan X, Liu D, Li $M$, Song $Y$, Z Zhu $X$, Sun $H$, Feng $T$, Guo $Z$, Ju A, Ge J, Dong $Y$, Sun W, Jiang $Y$, Wang J, Yan J, Yang H, Wang X, Gao GF, Yang R, Wang J, Yu J: A glimpse of streptococcal toxic shock syndrome from comparative genomics of $S$. suis 2 Chinese isolates. PLoS One 2007, 2:e315.

41. Allgaier A, Goethe R, Wisselink HJ, Smith HE, Valentin-Weigand P: Relatedness of Streptococcus suis isolates of various serotypes and clinical backgrounds as evaluated by macrorestriction analysis and expression of potential virulence traits. J Clin Microbiol 2001, 39:445-453.

42. Betriu C, Gomez M, Sanchez A, Cruceyra A, Romero J, Picazo JJ: Antibiotic resistance and penicillin tolerance in clinical isolates of group $B$ streptococci. Antimicrob Agents Chemother 1994, 38:2183-2186.

43. Pichichero ME, Casey JR: Systematic review of factors contributing to penicillin treatment failure in Streptococcus pyogenes pharyngitis. Otolaryngol Head Neck Surg 2007, 137:851-857.

44. Entenza JM, Caldelari I, Glauser MP, Francioli P, Moreillon P: Importance of genotypic and phenotypic tolerance in the treatment of experimental endocarditis due to Streptococcus gordonii. J Infect Dis 1997, 175:70-76.

45. Orman MA, Brynildsen MP: Establishment of a method to rapidly assay bacterial persister metabolism. Antimicrob Agents Chemother 2013, 57:4398-4409.

46. Luidalepp $H$, Joers $A$, Kaldalu $N$, Tenson $T$ : Age of inoculum strongly influences persister frequency and can mask effects of mutations implicated in altered persistence. J Bacteriol 2011, 193:3598-3605.

47. Bizzini A, Entenza JM, Moreillon P: Loss of penicillin tolerance by inactivating the carbon catabolite repression determinant $\mathrm{CcpA}$ in Streptococcus gordonii. J Antimicrob Chemother 2007, 59:607-615.

48. Bradely JJ, Mayhall CG, Dalton HP: Incidence and characteristics of antibiotic-tolerant strains of Staphylococcus aureus. Antimicrob Agents Chemother 1978, 13:1052-1057.

49. Sader HS, Flamm RK, Farrell DJ, Jones RN: Daptomycin activity against uncommonly isolated streptococcal and other gram-positive species groups. Antimicrob Agents Chemother 2013, 57:6378-6380.

50. Francois B, Gissot V, Ploy MC, Vignon P: Recurrent septic shock due to Streptococcus suis. J Clin Microbiol 1998, 36:2395

51. Bonifait L, Grignon L, Grenier D: Fibrinogen induces biofilm formation by Streptococcus suis and enhances its antibiotic resistance. App/ Environ Microbiol 2008, 74:4969-4972.

52. Olson ME, Ceri H, Morck DW, Buret AG, Read RR: Biofilm bacteria: formation and comparative susceptibility to antibiotics. Can J Vet Res 2002, 66:86-92.

53. Brisebois LM, Charlebois R, Higgins R, Nadeau M: Prevalence of Streptococcus suis in four to eight week old clinically healthy piglets. Can $J$ Vet Res 1990, 54:174-177.

54. Maclnnes II, Gottschalk M, Lone AG, Metcalf DS, Ojha S, Rosendal T, Watson SB, Friendship RM: Prevalence of Actinobacillus pleuropneumoniae, Actinobacillus suis, Haemophilus parasuis, Pasteurella multocida, and Streptococcus suis in representative Ontario swine herds. Can J Vet Res 2008, 72:242-248.

55. Amass SF, Wu CC, Clark LK: Evaluation of antibiotics for the elimination of the tonsillar carrier state of Streptococcus suis in pigs. J Vet Diagn Invest 1996, 8:64-67.

56. Smith $H E$, Veenbergen $V$, Van der Velde J, Damman M, Wisselink HJ, Smits MA: The cps genes of Streptococcus suis serotypes 1, 2, and 9: development of rapid serotype-specific PCR assays. J Clin Microbiol 1999, 37:3146-3152.

57. Schubert A, Zakikhany K, Schreiner M, Frank R, Spellerberg B, Eikmanns BJ, Reinscheid DJ: A fibrinogen receptor from group B Streptococcus interacts with fibrinogen by repetitive units with novel ligand binding sites. Mol Microbiol 2002, 46:557-569.

58. Rohde M, Muller E, Chhatwal GS, Talay SR: Host cell caveolae act as an entry-port for group A streptococci. Cell Microbiol 2003, 5:323-342.

59. Molinari G, Talay SR, Valentin-Weigand P, Rohde M, Chhatwal GS: The fibronectin-binding protein of Streptococcus pyogenes, Sfbl, is involved in the internalization of group A streptococci by epithelial cells. Infect Immun 1997, 65:1357-1363.

\section{doi:10.1186/1471-2180-14-120}

Cite this article as: Willenborg et al:: Characterization of multi-drug

tolerant persister cells in Streptococcus suis. BMC Microbiology 2014 14:120 